\section{Große Variabilität der Exazerbationsfrequenz bei COPD}

Han MLK et al. Frequency of exacerbations in patients with chronic obstructive pulmonary disease: an analysis of the SPIROMICS cohort. Lancet Respir Med 2017; 5: 619 - 626. doi:10.1016/S2213-2600(17)30207-2

Wenn Patienten mit einer chronisch obstruktiven Atemwegserkrankung (COPD) häufig Exazerbationen haben, geht dies mit einem Verlust an Lebensqualität und einer rascheren Verschlechterung der Lungenfunktion einher als bei Patienten ohne Exazerbationen. Deshalb werden in Therapiestudien meist Patienten mit mindestens zwei Exazerbationen in den letzten 12 Monaten rekrutiert. Diese plötzlichen Verschlechterungen treten aber nicht gleichmäßig auf.

Mei Lan K. Han von der Abteilung für Lungenheilkunde und Intensivmedizin des Universitätsklinikums Ann Arbor (Michigan, USA) und Koautoren analysierten Daten einer Kohorte von Patienten der „Subpopulations and Intermediate Outcome Measures in COPD Study“ (SPIROMICS), um longitudinal und prospektiv die Exazerbationen von Patienten im Alter von 40 bis 80 Jahren über mindestens 3 Jahre nachzuverfolgen. Sie unterteilten die 1.105 Patienten mit COPD, für die ausreichend lange Beobachtungsdaten vorlagen, entsprechend der jährlichen Frequenz der Exazerbationen in $\mathrm{Pa}$ tienten mit
- keiner Exazerbation in einem der Beobachtungsjahre,

- einer Exazerbation in jedem Jahr der Beobachtung,

- inkonsistentem Exazerbationsmuster (in manchen Jahren Exazerbationen, in anderen keine).

In einer schrittweisen logistischen Regressionsanalyse suchten sie nach Faktoren, die mit einer oder mehreren akuten Exazerbationen pro Jahr oder mit keiner Exazerbation über 3 Jahre assoziiert waren.

\section{Ergebnisse}

Von den 1.105 Patienten wiesen 538 (49\%) mindestens eine akute Exazerbation in den drei Beobachtungsjahren auf, 567 (51\%) keine einzige. Mindestens eine Exazerbation pro Jahr war bei 82 Patienten (7\%) dokumentiert, zwei und mehr akute Exazerbationen in jedem Jahr nur bei 23 (2\%). Ein inkonsistentes Muster von Exazerbationen war häufig (456 Patienten, 41\%). Das betraf insbesondere Patienten mit einem GOLD-Stadium 3 oder 4: 256 von insgesamt 456 dieser Patienten (56\%) wiesen ein solches Muster auf.

Die Regressionsanalyse zeigte, dass Patienten mit mindestens einer Exazerbation in jedem Jahr zum Zeitpunkt des Studieneinschlusses eine höhere COPDSymptomlast aufwiesen, früher bereits Exazerbationen erlitten hatten, im Spiral-CT deutlichere Zeichen eines pathologischen Befunds der kleinen Atemwege aufwiesen, und niedrigere Interleukin (IL)-15-Konzentrationen sowie höhere IL-
8-Konzentrationen hatten als diejenigen ohne akute Exazerbationen in der Beobachtungszeit.

FAZIT

Akute Exazerbationen bei Patienten mit COPD sind häufig, aber ihr Auftreten variiert bei den meisten $\mathrm{Pa}$ tienten von Jahr zu Jahr, betonen die Autoren. Die wenigsten Patienten haben innerhalb von drei Jahren zwei und mehr Exazerbationen pro Jahr. Statt der Exazerbationsrate können möglicherweise die beschriebenen Biomarker eher Hinweise auf das Exazerbationsrisiko bei individuellen $\mathrm{Pa}$ tienten geben. Bei seltenen Exazerbationen sind eher externe Trigger von Bedeutung, die schwer vorherzusagen sind.

Friederike Klein, München 